\title{
Shape coexistence in lead isotopes in the interacting boson model with a Gogny energy density functional
}

\author{
K. Nomura, ${ }^{1,{ }^{*}}$ R. Rodríguez-Guzmán, ${ }^{2,3}$ L. M. Robledo, ${ }^{4}$ and N. Shimizu ${ }^{5}$ \\ ${ }^{1}$ Institut für Kernphysik, Universität zu Köln, D-50937 Köln, Germany \\ ${ }^{2}$ Department of Physics and Astronomy, Rice University, Houston, Texas 77005, USA \\ ${ }^{3}$ Department of Chemistry, Rice University, Houston, Texas 77005, USA \\ ${ }^{4}$ Departamento de Física Teórica, Universidad Autónoma de Madrid, E-28049 Madrid, Spain \\ ${ }^{5}$ Center for Nuclear Study, University of Tokyo, Hongo, Bunkyo-ku, 113-0033 Tokyo, Japan
}

(Received 23 August 2012; published 17 September 2012)

\begin{abstract}
We investigate the emergence and evolution of shape coexistence in the neutron-deficient lead isotopes within the interacting boson model (IBM) plus configuration mixing with microscopic input based on the Gogny energy density functional (EDF). The microscopic potential-energy surface obtained from the constrained self-consistent Hartree-Fock-Bogoliubov method employing the Gogny-D1M EDF is mapped onto the coherent-state expectation value of the configuration mixing IBM Hamiltonian. In this way, the parameters of the IBM Hamiltonian are fixed for each of the three relevant configurations (spherical, prolate, and oblate) associated to the mean-field minima. Subsequent diagonalization of the Hamiltonian provides the excitation energy of the low-lying states and transition strengths among them. The model predictions for the $0^{+}$level energies and evolving shape coexistence in the considered lead chain are consistent both with experiment and with the indications of the Gogny-EDF energy surfaces.
\end{abstract}

DOI: 10.1103/PhysRevC.86.034322

PACS number(s): 21.10.Re, 21.60.Ev, 21.60.Fw, 21.60.Jz

\section{INTRODUCTION}

The atomic nucleus is a physical system that exhibits a rich variety of intrinsic geometrical shapes: spherical, prolate, and oblate. The coexistence and evolution of the different intrinsic shapes has been a major theme of interest in nuclear structure physics. It has been investigated extensively from both theoretical and experimental sides in the past decades [1-7].

In some specific regions of the Nuclide Chart, the energies of the three intrinsic geometrical shapes bunch together, leading to the spectacular coexistence of three $0^{+}$states (including the ground state) in even-even nuclei. Neutron-deficient lead isotopes present a nice example of the shape coexistence phenomena [2]: In the ${ }^{186,188} \mathrm{~Pb}$ nuclei, the presence of three low-lying $0^{+}$states and other additional experimental data strongly suggests the coexistence of spherical, prolate, and oblate shapes. In the context of the nuclear shell model [6-9], the emergence of low-lying excited $0^{+}$states is traced back to the proton particle-hole excitation across the $Z=82$ closed shell. The residual interaction between protons and neutrons is enhanced due to this cross-shell excitation, resulting in the lowering of the excited $0^{+}$states. In the vicinity of the $N=104$ midshell, the effect is strengthened and has a stronger impact on excitation energies. For the ${ }^{186} \mathrm{~Pb}$ nucleus, the three lowest $0^{+}$states are within a range of $700 \mathrm{keV}$ and the two intruder $0^{+}$ levels have the lowest excitation energy among the members of the $\mathrm{Pb}$ chain. The first excited $0^{+}$state is interpreted as a proton two-quasiparticle $\left(\pi \mathrm{h}_{9 / 2}\right)^{2}$ intruder configuration, while the second excited $0^{+}$state could be interpreted as a proton four-quasiparticle $\left(\pi \mathrm{h}_{9 / 2}\right)^{4}$ intruder configuration. These $0^{+}$ states correspond to oblate and prolate equilibrium shapes.

\footnotetext{
*nomura@ikp.uni-koeln.de
}

More quantitative results using large-scale shell-model calculations can only be obtained in lighter nuclei. However, for heavy nuclei including the lead isotopes the dimension of the shell-model configuration space becomes exceedingly large and a truncation strategy preserving the essential ingredients of the low-energy spectrum is required. The interacting boson model (IBM) [10] has been successfully used for describing the low-lying states of medium-heavy and heavy nuclei, and presents a severe truncation of the full shell-model space [11-13]. In this case, the building blocks are $s$ and $d$ bosons, which reflect the collective $J^{\pi}=0^{+}$and $2^{+}$pairs of valence nucleons, respectively [11-13].

Within the IBM, the description of intruder $0^{+}$states is based on the model by Duval and Barrett $[14,15]$. They proposed to mix the normal $(0 \mathrm{p}-0 \mathrm{~h})$ configuration, comprised of $N$ bosons, with intruder configurations comprised of $N+2 n(n \geqslant 1,2, \ldots)$ bosons, which takes into account the $2 n$-particle- $2 n$-hole excitation across the closed shell. In the case of $\mathrm{Pb}$ isotopes with three low-lying $0^{+}$levels, the model consists of three different Hamiltonians corresponding to $0 p-0 h, 2 p-2 h$, and $4 p-4 h$ configurations. The idea of configuration mixing in the IBM framework has been applied to spectroscopic analyses [9,16-19], algebraic features [20,21], and geometry and phases [22-24] associated with the shape coexistence observed in the lead and mercury region. In these studies, the parameters for the configuration mixing IBM Hamiltonian have been extracted from a fit to the experimental spectra and transition rates.

The different configurations of the shell model are related in the mean-field language to the minima of the corresponding mean-field deformation energy surface. The self-consistent mean-field method using microscopic energy density functionals (EDFs) currently provides an accurate and universal description of nuclear ground-state properties 
and low-energy collective excitations, including mass, density distributions, surface deformation, giant resonance, etc. The most popular EDFs can be of zero-range Skyrme [25], finite-range Gogny [26], as well as several parametrizations of the relativistic mean-field (RMF) Lagrangian [27,28]. The qualities and instabilities of the self-consistent description of shape coexistence, based on a series of Skyrme interactions, were examined in Ref. [29]. On the other hand, the so called NL-SC (Shape Coexistence) RMF parametrization has been tailored to describe the pronounced shape coexistence in $\mathrm{Pb}$, $\mathrm{Hg}$, and Pt isotopes [30]. The Nilsson-Strutinsky method has also been used to study the neutron-deficient $\mathrm{Pb}$ and $\mathrm{Hg}$ isotopes [31].

At the mean-field level, however, important symmetries of the system are spontaneously broken. Therefore, to describe the spectroscopic properties of a given nucleus, one needs a systematic treatment of the dynamical effects associated with the restoration of the broken symmetries and fluctuations in the collective coordinates. It is then necessary to project the mean-field solutions onto states with good symmetry quantum numbers and mix the different configurations. Configuration mixing calculations, in the spirit of the generator coordinate method (GCM), have been performed for both lead and mercury nuclei, based on Skyrme [32,33] and Gogny [34-36] EDFs.

A sound approximation to the full GCM configuration mixing calculation is represented by the solution of a fivedimensional collective Hamiltonian. Both vibrational and rotational mass parameters are obtained, from mean-field calculations, as functions of the quadrupole collective variables. The collective potential is then taken as the total-energy surface resulting from the mean-field approximation from which the zero-point energies associated with the rotational and vibrational motions are subtracted [37,38]. This method can be also used for the description of shape coexistence phenomena based on arbitrary EDFs, e.g., using the Gogny-D1S functional for $\mathrm{Hg}$ isotopes [39].

More recently a comprehensive way of deriving the parameters of the IBM Hamiltonian has been introduced [40]. By mapping the potential-energy surface (PES), obtained within the constrained self-consistent mean-field method with a given $\mathrm{EDF}$, onto the expectation value of the corresponding IBM Hamiltonian, the energy spectra and electromagnetic transition rates have been computed. This method has been successfully applied to various shape phenomena, including vibrational and $\gamma$-unstable [41] as well as rotational deformed [42] nuclei, to prolate-oblate shape transitions [43], and to the study of the fingerprints of triaxiality [44].

In this paper we extend the method of [40] to take into account configuration mixing within the IBM. We will show how the parameters of the configuration mixing IBM Hamiltonian can be determined without a fit to the experiment by using the microscopic input provided by mean-field energy surfaces in an appropriate way. Using this method, we are able to describe the emergence and evolution of shape coexistence. Our method is applied to the neutron-deficient $\mathrm{Pb}$ isotopes since the existence of three minima in some of them represents a quite stringent test of the model. Moreover, they are well studied both experimentally and theoretically, offering us the possibility to benchmark our method with other proposals. Concerning the mean-field calculation, we use the GognyD1M [45] functional that was originally fitted to binding energies and radii. It has also shown good spectroscopic properties as already exemplified in previous studies [46-48], where it has been shown that D1M keeps essentially the same predictive power as the standard Gogny-D1S EDF [49].

The paper is organized as follows. In Sec. II, a brief review of the configuration mixing within the IBM and the geometrical interpretation is given. The mapping of the microscopic PES to the IBM one with configuration mixing is described and the way to extract the IBM parameters is discussed in Sec. III. In Sec. IV, the results of the diagonalization of the IBM Hamiltonian including energy-level systematics, the detailed level scheme, and the $B(\mathrm{E} 2)$ transition strength values for specific nuclei and the evolution of the spectroscopic quadrupole moment in the considered $\mathrm{Pb}$ chain are presented. Finally, Sec. V is devoted to the conclusions and work perspectives.

\section{DESCRIPTION OF THE MODEL}

We start with the self-consistent constrained Hartree-FockBogoliubov (HFB) approximation based on the Gogny-D1M EDFs. As constraints we use the mass quadrupole moments associated with the quadrupole deformation variables $\beta$ and $\gamma$ of the geometrical collective model [50]. For a given set of collective coordinate variables $q=(\beta, \gamma)$, HFB calculations are performed to obtain the potential-energy surface (PES) given by the HFB total energy denoted as $E^{\mathrm{HFB}}(\beta, \gamma)$ (for details the reader is referred to [51]). Note that, in some studies dealing with the five-dimensional collective Hamiltonian obtained from EDF calculations [37,38], the PES is regarded as the total energy obtained after subtraction of the rotational and vibrational zero-point energies to the HFB energy. In our model the PES is simply the HFB energy and no zero-point energy corrections are considered. A typical example of such PESs is shown in Fig. 1, where the Gogny-D1M PESs in the $(\beta, \gamma)$ plane are given for the nuclei ${ }^{182-192} \mathrm{~Pb}$. The Gogny-D1M EDF calculation produces a remarkable triple minima in ${ }^{184-190} \mathrm{~Pb}$ nuclei, where each local minimum is well isolated from each other. In all the considered lead nuclei, the spherical minimum is always present while a prolate minimum develops from ${ }^{182} \mathrm{~Pb}$ to ${ }^{186} \mathrm{~Pb}$, so does the oblate one. The prolate minimum becomes less significant from ${ }^{188} \mathrm{~Pb}$ to ${ }^{190} \mathrm{~Pb}$ and finally disappears in ${ }^{192} \mathrm{~Pb}$. Within our model the Gogny-D1M EDF PES for an individual nucleus is mapped onto the corresponding IBM PES (to be discussed below), as shown in Fig. 1 in the panels to the right of the Gogny ones.

Let us turn to the IBM description of shape coexistence. In the present study, we consider the proton-neutron version of the IBM (usually referred as IBM-2) [11,13] since it takes into account proton excitations more explicitly than the original version of the IBM (IBM-1), which does not distinguish between proton and neutron degrees of freedom. The IBM-2 comprises the neutron (proton) $s_{v}\left(s_{\pi}\right)$ and $d_{\nu}\left(d_{\pi}\right)$ bosons, reflecting the neutron (proton) $J^{\pi}=0^{+}$and $2^{+}$collective pairs of valence nucleons [11-13]. The number of neutron (proton) bosons, denoted as $N_{v}\left(N_{\pi}\right)$, equals the number of neutron (proton) pairs outside the inert core. 

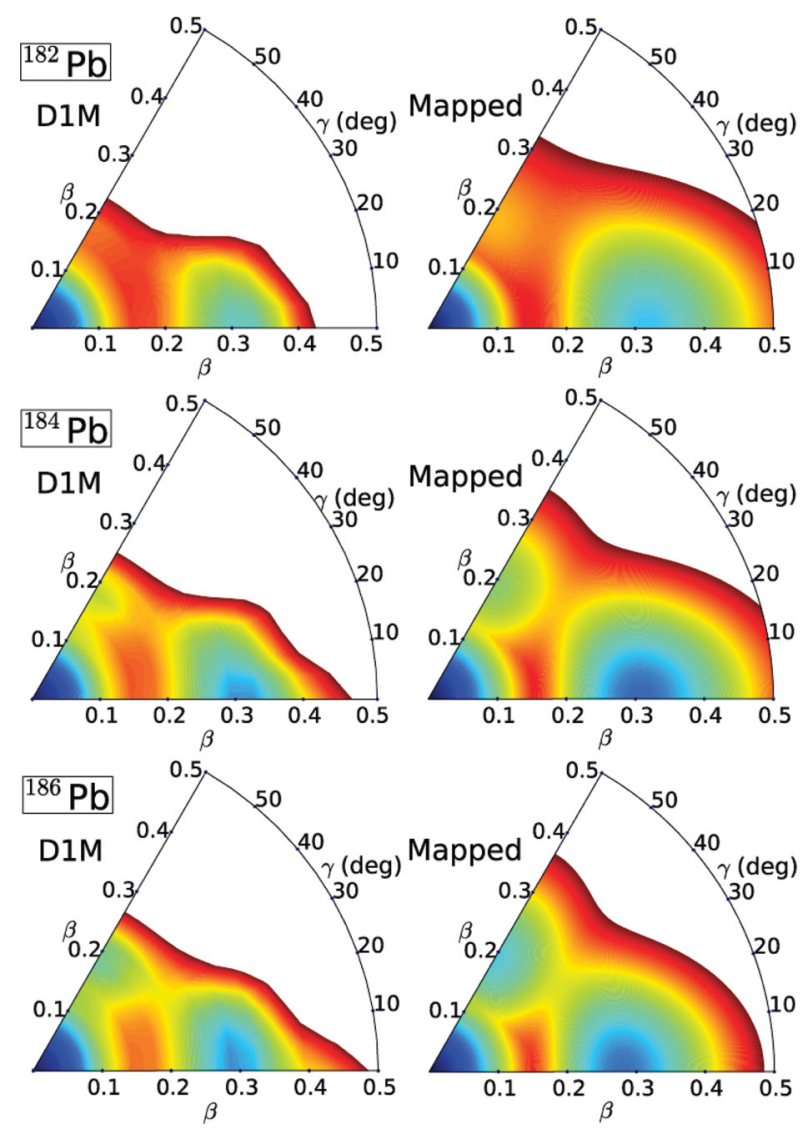
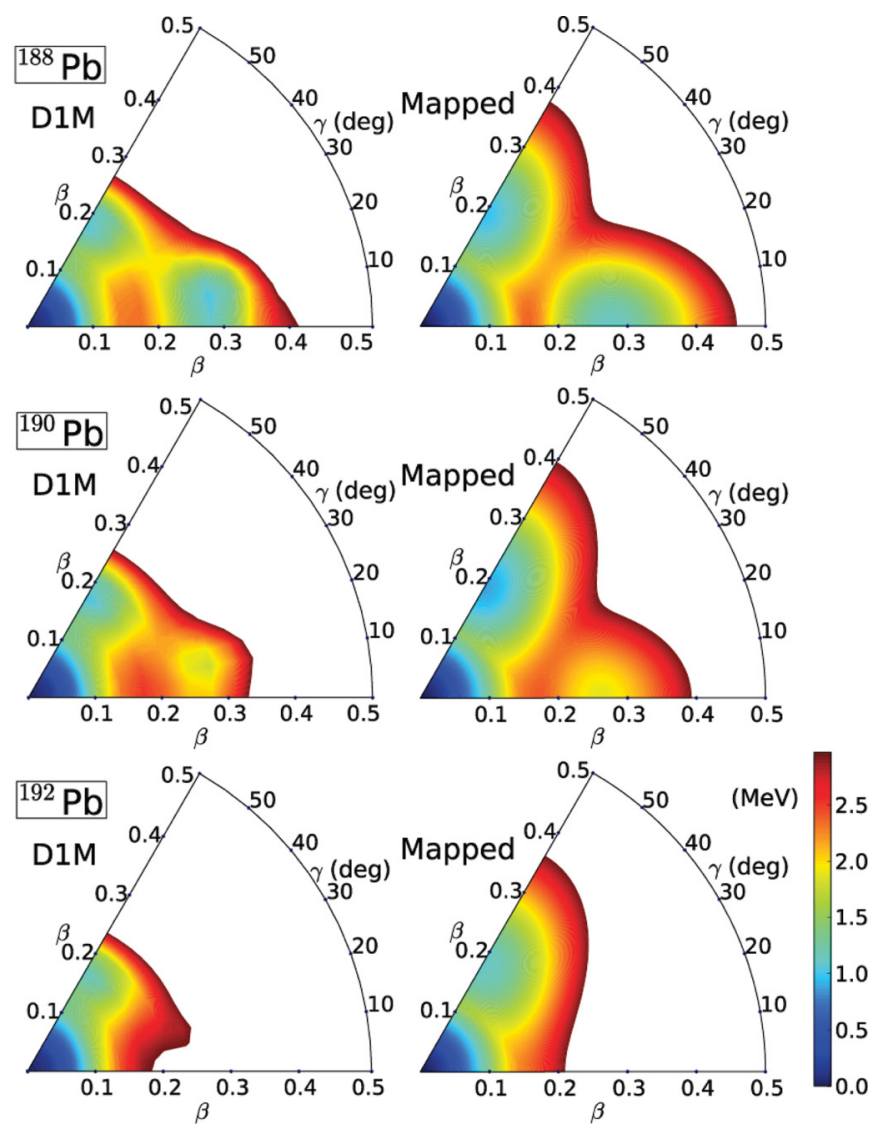

FIG. 1. (Color online) Contour plots of the PESs as a function of the deformation parameters $\beta$ and $\gamma$ given for the ${ }^{182-192} \mathrm{~Pb}$ isotopes. The plots denoted by D1M correspond to the microscopic mean-field calculation with the Gogny-D1M EDF. The ones denoted by Mapped correspond to the mapped PESs used in fitting the IBM parameters. The color scale ranges from 0 (the mean-field ground state) to $3 \mathrm{MeV}$.

To describe a system consisting of three different intrinsic shapes, the Hilbert space is expressed as a direct sum of the orthogonal subspaces for the normal $(0 \mathrm{p}-0 \mathrm{~h})$ and the two intruder (2p-2h and $4 \mathrm{p}-4 \mathrm{~h})$ configurations $[14,15]$. The Hamiltonian of the system is written as

$$
\begin{aligned}
\hat{H}= & \hat{P}_{0} \hat{H}_{0} \hat{P}_{0}+\hat{P}_{2}\left(\hat{H}_{2}+\Delta_{2}\right) \hat{P}_{2} \\
& +\hat{P}_{4}\left(\hat{H}_{4}+\Delta_{4}\right) \hat{P}_{4}+\hat{H}_{\text {mix }}^{02}+\hat{H}_{\text {mix }}^{24},
\end{aligned}
$$

where the $\hat{H}_{i}(i=0,2,4)$ represent the Hamiltonians for the $i \mathrm{p}-i \mathrm{~h}$ configurations associated with the different intrinsic shapes and $\hat{H}_{\text {mix }}^{02}\left(\hat{H}_{\text {mix }}^{24}\right)$ are the interaction terms mixing the $0 \mathrm{p}-0 \mathrm{~h}(2 \mathrm{p}-2 \mathrm{~h})$ and the $2 \mathrm{p}-2 \mathrm{~h}(4 \mathrm{p}-4 \mathrm{~h})$ subspaces. The operators $\hat{P}_{i}$ are projectors onto the $i \mathrm{p}-i \mathrm{~h}$ configuration spaces and finally the $\Delta_{i}(i=2,4)$ parameters represent the energies needed to excite protons across the $Z=82$ shell, which will be detailed later.

We employ the Hamiltonian $\hat{H}_{i}$ written as

$$
\hat{H}_{i}=\epsilon_{i} \hat{n}_{d}+\kappa_{i} \hat{Q}_{\pi}^{\chi_{\pi, i}} \cdot \hat{Q}_{\nu}^{\chi_{v, i}},
$$

where the first term $\hat{n}_{d}=\hat{n}_{d \pi}+\hat{n}_{d v}$ represents the $d$-boson number operator while the second one is the quadrupolequadrupole interaction between proton and neutron bosons. The quadrupole operator is defined as $\hat{Q}_{\rho}^{\chi_{\rho, i}}=s_{\rho}^{\dagger} \tilde{d}_{\rho}+d_{\rho}^{\dagger} s_{\rho}+$ $\chi_{\rho, i}\left[d_{\rho}^{\dagger} \tilde{d}_{\rho}\right]^{(2)}(\rho=\pi, v)$. In this case $\epsilon_{i}, \kappa_{i}$, and $\chi_{\rho, i}$ are parameters. The Hamiltonian in Eq. (2) is taken in its simplified form in order to reduce the number of parameters that are not directly determined from the PES. It keeps, however, the essential aspects of a more general IBM-2 Hamiltonian.

The mixing interaction terms $\hat{H}_{\text {mix }}^{i-2 i}(i=2,4)$ are defined as

$$
\hat{H}_{\text {mix }}^{i-2 i}=\omega_{1}^{i-2 i}\left(s_{\pi}^{\dagger} s_{\pi}^{\dagger}+s_{\pi} s_{\pi}\right)+\omega_{2}^{i-2 i}\left(d_{\pi}^{\dagger} \cdot d_{\pi}^{\dagger}+\tilde{d}_{\pi} \cdot \tilde{d}_{\pi}\right),
$$

where $\omega_{1}^{i-2 i}$ and $\omega_{2}^{i-2 i}$ stand for the mixing strengths.

In a shell-model picture, the proton $2 \mathrm{p}-2 \mathrm{~h}$ excitation across the closed shell $Z=82$ creates one particle and one hole pair in the $Z=82-126$ and 50-82 major shells, respectively. Since the IBM normally does not distinguish between particle and hole states, the $2 n$-particle- $2 n$-hole configuration comprises $2 n$ additional proton bosons, and hence the model contains $N_{\nu}$ neutron bosons and $N_{\pi}+2 n$ proton bosons. For the considered ${ }^{182-192} \mathrm{~Pb}$ nuclei, the doubly magic systems ${ }^{164} \mathrm{~Pb}$ and ${ }^{208} \mathrm{~Pb}$ are assumed to be the inert cores. As a consequence, the proton boson numbers are $N_{\pi}=0,2$, and 4 for regular, $2 \mathrm{p}-2 \mathrm{~h}$, and $4 \mathrm{p}-4 \mathrm{~h}$ configurations, respectively, while $N_{v}$ varies between 8 and 11 .

A given IBM Hamiltonian can be related to the geometrical model by the coherent-state framework [52]. The coherent state $|\Phi\rangle$ represents the intrinsic wave function of the boson 
system, and is written, up to a normalization factor, as

$$
|\Phi\rangle=\prod_{\rho=\pi, \nu}\left(s_{\rho}^{\dagger}+\sum_{\mu=-2}^{2} a_{\rho \mu} d_{\rho \mu}^{\dagger}\right)^{N_{\rho}}|0\rangle,
$$

where the coefficients $a_{\rho \mu}$ are given by $a_{\rho 0}=\beta_{\rho} \cos \gamma_{\rho}$, $a_{\rho \pm 1}=0$, and $a_{\rho \pm 2}=\frac{1}{\sqrt{2}} \beta_{\rho} \sin \gamma_{\rho}$. Here the parameters $\beta_{\rho}$ and $\gamma_{\rho}$ represent the axially symmetric and the triaxial deformations for neutrons $(\rho=v)$ and protons $(\rho=\pi)$, respectively. For simplicity we assume $\beta_{v}=\beta_{\pi}=\beta_{B}$ and $\gamma_{v}=\gamma_{\pi}=\gamma_{B}$. The $\beta$ parameter for the IBM is proportional to the one in the geometrical model. The proportionality coefficient is significantly larger than 1 due to the difference in the size of the model spaces [52]. On the other hand, the $\gamma$ variable can be the same for the IBM and the geometrical model. The PES for the IBM system of interest is given analytically as an energy expectation value of the coherent state [52].

The geometrical interpretation of the configuration mixing IBM was provided by Frank et al. [22]. The coherent state in Eq. (4) for a single configuration should be extended to be a direct sum of the coherent state for each configuration. The PES for the configuration mixing IBM is obtained as the lowest eigenvalue of the following $3 \times 3$ matrix [22]:

$$
E(\beta, \gamma)=\left(\begin{array}{ccc}
E_{0}(\beta, \gamma) & \Omega_{02}(\beta) & 0 \\
\Omega_{02}(\beta) & E_{2}(\beta, \gamma)+\Delta_{2} & \Omega_{24}(\beta) \\
0 & \Omega_{24}(\beta) & E_{4}(\beta, \gamma)+\Delta_{4}
\end{array}\right),
$$

where the $E_{i}(\beta, \gamma)(i=0,2,4)$ in the diagonal part stands for the expectation value of the Hamiltonian $\hat{H}_{i}$ :

$$
\begin{aligned}
& E_{i}(\beta, \gamma) \\
& =\frac{\epsilon_{i}\left(N_{\nu}+N_{\pi, i}\right) \beta_{B, i}^{2}}{1+\beta_{B, i}^{2}}+\kappa_{i} N_{\nu} N_{\pi, i} \frac{\beta_{B, i}^{2}}{\left(1+\beta_{B, i}^{2}\right)^{2}} \\
& \quad \times\left[4-2 \sqrt{\frac{2}{7}}\left(\chi_{\nu, i}+\chi_{\pi, i}\right) \beta_{B, i} \cos 3 \gamma+\frac{2}{7} \chi_{\nu, i} \chi_{\pi, i} \beta_{B, i}^{2}\right] .
\end{aligned}
$$

Here $\beta_{B}^{i}=C_{\beta, i} \beta$, with $C_{\beta, i}$ being the proportionality coefficient of the $\beta$ variable defined for the different mean-field minima associated with each configuration $i \mathrm{p}-i \mathrm{~h}$, and $N_{\pi, i}$ denotes the proton boson number in the $i \mathrm{p}-i \mathrm{~h}$ configuration. The nondiagonal entries $\Omega_{i-2 i}(\beta)(i=2,4)$ represent the expectation values of the mixing interactions $\hat{H}_{\text {mix }}^{i-2 i}$, given as

$$
\begin{aligned}
\Omega_{i-2 i}(\beta)= & \frac{\sqrt{N_{\pi, i}\left(N_{\pi, i}-1\right)}}{1+\beta_{B, i}^{2}}\left(\omega_{1}^{i-2 i}+\omega_{2}^{i-2 i} \beta_{B, i}^{2}\right) \\
& \times\left(\frac{1+\beta_{B, i-2} \beta_{B, i}}{\sqrt{\left(1+\beta_{B, i-2}^{2}\right)\left(1+\beta_{B, i}^{2}\right)}}\right)^{N_{\nu}+N_{\pi, i-2}}
\end{aligned}
$$

Each of the microscopic PESs, presented in Fig. 1, is mapped onto the corresponding IBM PES, i.e., the lowest eigenvalue of the matrix in Eq. (5). Since the three local minima are well separated from each other, a set of parameters for each configuration is determined independently from the others. First, the 0p-0h configuration is assigned to the mean-field minimum with the smallest deformation. Then the $2 \mathrm{p}-2 \mathrm{~h}$ configuration is assigned to the minimum with the second larger quadrupole deformation. Likewise the $4 \mathrm{p}-4 \mathrm{~h}$ configuration is associated with the minimum with the third larger quadrupole deformation. For each configuration, the parameters $\epsilon_{i}, \kappa_{i}$, $\chi_{\nu, i}, \chi_{\pi, i}$, and $C_{\beta, i}$ in $E_{i}(\beta, \gamma)$ of Eq. (6) are determined, using the method of [41], so that the topologies, i.e., curvatures in both $\beta$ and $\gamma$ directions, around the corresponding minima are reproduced. For ${ }^{186} \mathrm{~Pb}$, for instance, the Hamiltonians for $0 \mathrm{p}-0 \mathrm{~h}, 2 \mathrm{p}-2 \mathrm{~h}$, and $4 \mathrm{p}-4 \mathrm{~h}$ configurations are assigned to spherical $(\beta=0)$, oblate $(\beta \approx-0.2)$, and prolate $(\beta \approx+0.3)$ minima, respectively. Since the number of proton bosons $N_{\pi}$ is zero for all the considered $\mathrm{Pb}$ nuclei, the second term in Eq. (2) vanishes, and the parameters $\kappa_{0}, \chi_{\nu, 0}$, and $\chi_{\pi, 0}$ can be set to zero. Therefore, in the present study, the $0 \mathrm{p}-0 \mathrm{~h}$ configuration always represents a pure $\mathrm{U}(5)$ limit of the IBM [10].

The $\Delta_{i}$ parameters in Eq. (5) are constants depending on the nucleus and they are fixed so that the energy difference between the mean-field spherical and intruder configurations is reproduced. These energy differences between mean-field minima are denoted as $\delta E_{i}=E^{\mathrm{HFB}}\left(\beta_{\min }^{i}, \gamma_{\min }^{i}\right)-E^{\mathrm{HFB}}\left(\beta_{\min }^{0}, \gamma_{\min }^{0}\right)$ with $\left(\beta_{\min }^{i}, \gamma_{\min }^{i}\right)$ being the coordinates that give the minimum for each of the $i \mathrm{p}-i$ h configurations in the HFB PES. These quantities should be in reasonable agreement with the observed $0^{+}$excitation energies.

However, the values of the $\Delta_{i}$ derived from the estimation above should not be used in the spectroscopic calculations with the Hamiltonian of Eq. (1), i.e., different values of $\Delta_{i}$ should be used in Eqs. (1) and (5). From the original definition, the $\Delta_{i}(i=2$ or 4$)$ represents the offset energy added to the eigenenergies of the $i \mathrm{p}-i \mathrm{~h}$ Hamiltonian so that its ground-state $0^{+}$energy exceeds that of the normal configuration by an amount that is roughly equal to the observed excited $0^{+}$energy and hence to $\delta E_{i}$. More explicitly (cf. Appendix C of [15]),

$$
E_{i}\left(0^{+}\right)+\Delta_{i}=E_{0}\left(0^{+}\right)+\delta E_{i},
$$

where $E_{i}\left(0^{+}\right)$represents the lowest (ground-state) $0^{+}$eigenvalue of the $i \mathrm{p}-i \mathrm{~h}$ Hamiltonian in Eq. (2). Note that the amount of energy gained by the mixing between normal and intruder configurations is much smaller than the typical range of $\Delta_{i}$ values and is considered negligible in this rough estimate. In the considered $\mathrm{Pb}$ isotopes, since there is no deformation-driving term in the Hamiltonian $\hat{H}_{0}$, the $E_{0}\left(0^{+}\right)$ energy is always equal to zero for the $0 \mathrm{p}-0 \mathrm{~h}$ configuration. The lowest $0^{+}$eigenenergy comprises the energy gained through the deformation at the mean-field level (equivalent to the depth of the minimum in the PES) and the extra correlation energy arising from quantum effects beyond the mean field. The $\Delta_{i}$ values determined solely by looking at the PES do not take into account this quantum correlation energy and hence are too small to describe correct spectroscopic tendencies consistent with the indications of the microscopic PESs.

Let us consider, for example, the nucleus ${ }^{186} \mathrm{~Pb}$. The $\Delta_{2}$ value derived from the PES, to be used in Eq. (5), is 4.014 MeV. Nevertheless, with this value, the intruder $0^{+}$state becomes the ground state after the mixing. This is apparently 
not consistent with empirical facts or with the indication of the microscopic PES. Since the $2 \mathrm{p}-2 \mathrm{~h}$ configuration gives $E_{2}\left(0^{+}\right)=-3.676 \mathrm{MeV}$, to reproduce $\delta E_{2}=1.208 \mathrm{MeV}$ the $\Delta_{2}$ value to be used in Eq. (1) should amount to $\Delta_{2}=$ $1.208-(-3.676)=4.884 \mathrm{MeV}$. The difference between the two $\Delta_{2}$ values $(=0.870 \mathrm{MeV})$, identified as the quantum correlation energy that the $2 \mathrm{p}-2 \mathrm{~h}$ configuration gains through the diagonalization, seems so sizable as to change the conclusion. Therefore, for the spectroscopic calculations with the Hamiltonian of Eq. (1), we propose to use the formula in Eq. (8) to take into account the necessary quantum correlation effects. Also the $\Delta_{i}$ in Eq. (1) can be related to the ones in Eq. (5) by replacing $E_{i}\left(0^{+}\right)$in Eq. (8) by the deformation energy $E_{i}\left(\beta_{\min }^{i}, \gamma_{\min }^{i}\right)$, and vice versa. The uncertainty in the parameters relevant to the configuration mixing has also been pointed out in Ref. [22], where the PES of the configuration mixing IBM-1 Hamiltonian for lead nuclei was analyzed. Although the parameters of the Hamiltonian give a good description of the spectroscopy, only two (spherical and prolate) minima remain after configuration mixing in the ${ }^{186} \mathrm{~Pb}$ nucleus [22]. This result seems to support our finding that the $\Delta_{i}$ values to be used in spectroscopic calculations may not at the same time give the IBM mapped PES similar in topology to the mean-field PES.

To perform a fully consistent mapping of $\Delta_{i}$ in the present framework, the addition of some interaction term between like neutron bosons, such as of the $\kappa_{v} \hat{Q}_{\nu} \cdot \hat{Q}_{\nu}$ type, to the $0 \mathrm{p}-0 \mathrm{~h}$ Hamiltonian may solve the problem. The reason is that such a term drives deformation and provides the energy which could compensate for the quantum correlation energy the intruder configuration gains. In fact, if one tries to put $\kappa_{\nu} \hat{Q}_{\nu} \cdot \hat{Q}_{\nu}$ with the realistic interaction strength $\kappa_{v}=-0.013 \mathrm{MeV}$ in the mapped Hamiltonian $\hat{H}_{0}$ in Eq. (2) for ${ }^{186} \mathrm{~Pb}$, the $0 \mathrm{p}-0 \mathrm{~h}$ configuration gives $E_{0}\left(0^{+}\right)=-0.870 \mathrm{MeV}$, which is exactly the same as the correlation energy gained in the $2 \mathrm{p}-2 \mathrm{~h}$ configuration. Nevertheless, since the microscopic GognyD1M PES suggests a purely spherical minimum for the normal configuration, it is practically not possible to determine the strength parameter for such an additional interaction term. Another possible solution which could work out is to map the angular momentum projected PES onto the corresponding IBM PES. This could represent an interesting work for the future which is out of the scope of the present paper.

The nondiagonal matrix elements, $\Omega_{i-2 i}(\beta)$ in Eq. (7), concern the barrier between the mean-field minima but are only minor as compared to the diagonal parts in Eq. (6). Therefore, the parameter $\omega^{i-2 i}$ can be introduced only perturbatively and is determined so that the barrier height for two different minima in the microscopic PES is reproduced. For the sake of simplicity, we assume $\omega_{1}^{i-2 i}=\omega_{2}^{i-2 i} \equiv \omega^{i-2 i}$.

\section{MAPPED IBM POTENTIAL-ENERGY SURFACES AND DERIVED PARAMETERS}

The mapped IBM and the microscopic Gogny-D1M HFB PESs are plotted in Fig. 1 for the nuclei ${ }^{182-192} \mathrm{~Pb}$. In the case of ${ }^{192} \mathrm{~Pb}$ the HFB approximation suggests two minima, and therefore only the $0 \mathrm{p}-0 \mathrm{~h}$ and $2 \mathrm{p}-2 \mathrm{~h}$ configurations are mixed in this nucleus. The location, relative energy differences, as well as the energy barriers between the coexisting minima in the microscopic PESs are reproduced rather well in the mapped IBM PESs. Note that, due to the limited number of bosons, the mapped PESs are generally flat along the oblate axis. Although very shallow triaxial minima at $\gamma \approx 10^{\circ}$ are displayed in the HFB PESs of ${ }^{188,190} \mathrm{~Pb}$, in the mapped IBM PESs such minima are approximated by axial ones. As a result, some deviations of the barrier heights between the oblate and prolate minima occur for these nuclei. In order to describe the detailed energy systematics of the quasi- $\gamma$ band, a boson three-body term [44] is required which is, however, out of the scope of the present work.

The IBM parameters, derived for the considered isotopes ${ }^{182-192} \mathrm{~Pb}$, are displayed in Fig. 2. Consistent with the evolution of the topology in the PESs shown in Fig. 1, no rapid change
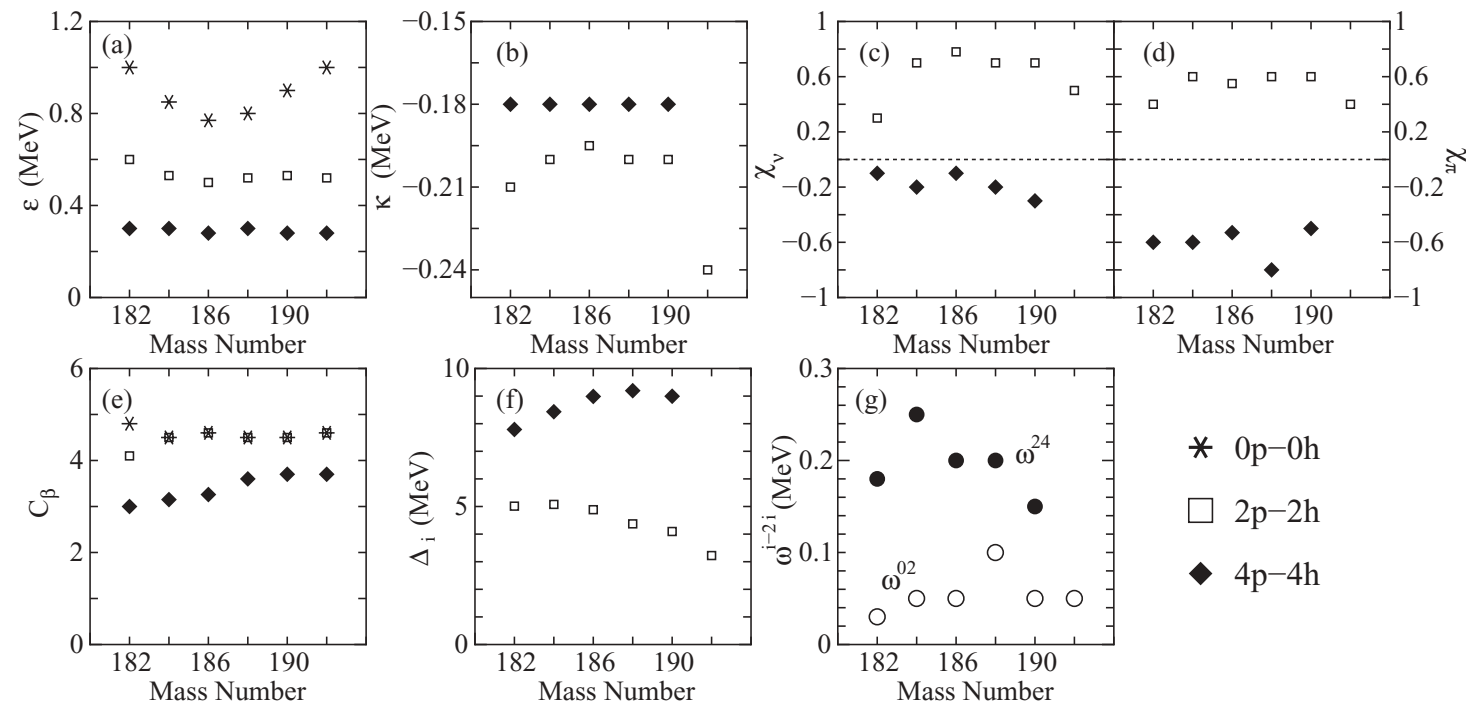

FIG. 2. Derived IBM parameters (a) $\epsilon_{i}$, (b) $\kappa_{i}$, (c) $\chi_{v, i}$, (d) $\chi_{\pi, i}$, (e) $C_{\beta, i}$, (f) $\Delta_{i}$, and (g) $\omega^{i-2 i}$ for the considered ${ }^{182-192} \mathrm{~Pb}$ nuclei as functions of mass number $A$. Figure legends for panels (a) through (f) are shown in the right-hand side of panel (g). 
with mass number is observed in these parameters. The comparison between the $\epsilon$ parameters, for a given nucleus, in Fig. 2(a) reveals that $\epsilon_{0}$ is the largest, $\epsilon_{4}$ is the smallest, and the $\epsilon_{2}$ value is always in between them. On the other hand, as a function of the mass number, $\epsilon_{0}$ looks parabolic with respect to the midshell nucleus ${ }^{186} \mathrm{~Pb}$, while $\epsilon_{2}$ and $\epsilon_{4}$ remain almost constant. Let us stress that these boson number dependencies are consistent with the earlier phenomenological (see [10] and references are therein) and microscopic [12,53] IBM-2 studies on collective structural evolution. The parameter $\kappa_{2}$ is, in general, larger than $\kappa_{4}$ as the model space of the latter contains a larger number of bosons. As functions of the mass number the $\chi$ parameters, shown in Figs. 2(c) and 2(d), also display a weak dependence. Nevertheless, the sign of $\chi_{v}$ is always opposite to the one of $\chi_{\pi}$. Their sum $\chi_{v}+\chi_{\pi}$ is positive (negative) for the oblate (prolate) $2 \mathrm{p}-2 \mathrm{~h}(4 \mathrm{p}-4 \mathrm{~h})$ shapes. The $C_{\beta}$ value (i.e., the scale factor for the $\beta$ variable) does not change too much. This parameter is determined from the position of the axial minimum and the curvature along the $\beta$ axis. Actually, the location of each axial minimum in the HFB PES in Fig. 1 remains almost the same in the corresponding IBM PES.

Figures 2(f) and 2(g) show the energy offset $\Delta_{i}$, defined in Eq. (8), and the mixing parameters for the Hamiltonian $\hat{H}_{\text {mix }}^{i-2 i}$, respectively. The magnitudes of both $\omega^{02}$ and $\omega^{24}$ are notably larger than those used in some fitted calculations within the configuration mixing IBM-1 model $[17,18]: \omega^{02} \approx$ $10 \mathrm{keV}$ and $\omega^{24} \approx 20-30 \mathrm{keV}$ in the latter studies, while we have obtained $\omega^{02} \approx 50 \mathrm{keV}$ and $\omega^{24} \approx 200 \mathrm{keV}$. The present $\omega^{24}$ value, which is particularly larger than the one derived from phenomenology, implies that our microscopic EDF approximation suggests a complex topology of the mean-field PESs in the studied lead isotopes in the $\gamma$ direction. Therefore, it may require a mixing between the two intruder configuration spaces stronger than estimated from the pure fitting calculations. In particular, the mixing between the regular and $2 \mathrm{p}-2 \mathrm{~h}$ configurations seems to be quite large in the case of ${ }^{188} \mathrm{~Pb}$.

The offset energy $\Delta_{i}$, depicted in Fig. 2(g), roughly amounts to 4 and $8 \mathrm{MeV}$ for the $2 \mathrm{p}-2 \mathrm{~h}$ and $4 \mathrm{p}-4 \mathrm{~h}$ configurations, respectively. These values are approximately twice as large as the ones obtained in the IBM-1 phenomenology [18]. One sees from Eq. (8) that a larger $\Delta_{i}$ energy is needed when the $0^{+}$ eigenenergy of the intruder configuration is sufficiently large in magnitude compared to the $0^{+}$energy of the normal configuration. The quadrupole-quadrupole interaction for the intruder configuration appears to be stronger in the present mapped IBM system than it is in the IBM-1 phenomenology. The intruder configuration gains a large amount of energy, giving rise to remarkable differences between our $\Delta_{i}$ values and the phenomenological $[17,18]$ results. In fact, the derived $\kappa_{2}$ and $\kappa_{4}$ values are larger in magnitude than those extracted from the fit. This may be due to the fact that the microscopic Gogny-D1M calculation (see Fig. 1) provides a pronounced minimum.

\section{SPECTROSCOPIC RESULTS}

Having determined all the parameters required by the IBM Hamiltonian in Eq. (1) for each individual nucleus, the energy spectra and transition rates are calculated by diagonalizing the Hamiltonian within the enlarged model space consisting of the direct sum of the $0 \mathrm{p}-0 \mathrm{~h}$, the $2 \mathrm{p}-2 \mathrm{~h}$, and the $4 \mathrm{p}-4 \mathrm{~h}$ subspaces. The results shown below are obtained without any fit to the experimental data, but only from the Gogny-D1M HFB approximation and the mapping procedure described above.

We have performed a diagonalization of the mapped IBM-2 Hamiltonian in the so-called boson $m$-scheme basis. The eigenfunction for each excited state gives rise to various spectral observables. In particular, the E2 transition rates and the spectroscopic quadrupole moments are important quantities by which one can gauge the emergence and the evolution of the coexistence and competition between different shapes in the considered isotopes. For the E2 operator $\hat{T}^{(\mathrm{E} 2)}$, we use the boson quadrupole operator $\hat{Q}_{\rho}^{\chi_{\rho, i}}$, where the same parameter $\chi_{\rho, i}$ as the one used in diagonalization is used, based on the idea of Casten and Warner for the IBM-1 case [54]. Within the configuration mixing IBM framework this E2 operator can be written as $[14,15]$

$$
\hat{T}^{(\mathrm{E} 2)}=\sum_{\rho, i} e_{\rho, i} \hat{P}_{i} \hat{Q}_{\rho}^{\chi_{\rho, i}} \hat{P}_{i},
$$

where $e_{\rho, i}$ represents the proton and neutron boson effective charges for each configuration. For simplicity, these charges are assumed to be the same (i.e., $e_{\nu, i}=e_{\pi, i} \equiv e_{i}$ ). For the effective charges, we have adopted the values given in Ref. [18] (i.e., $e_{0}=0.110, e_{2}=0.140$, and $e_{4}=0.170 e \mathrm{~b}$ ). The effective charge should, in principle, be determined by taking into account core polarization effects. Such an effect could be renormalized in the effective charges used here, while a fully microscopic derivation of the boson effective charge still represents an interesting open problem. With all this in mind, the reduced $\mathrm{E} 2$ transition $B\left(\mathrm{E} 2 ; J \rightarrow J^{\prime}\right)$ between states with spins $J$ and $J^{\prime}$ can be written as

$$
B\left(\mathrm{E} 2 ; J \rightarrow J^{\prime}\right)=\frac{1}{2 J+1}\left|\left\langle J^{\prime}|| \hat{T}^{(\mathrm{E} 2)}|| J\right\rangle\right|^{2},
$$

where $|J\rangle$ and $\left|J^{\prime}\right\rangle$ represent the wave functions of the initial and the final states with angular momenta $J$ and $J^{\prime}$, respectively.

The spectroscopic quadrupole moment $Q^{(s)}(J)$ for the state with spin $J$ is given by

$$
Q^{(s)}(J)=\sqrt{\frac{16 \pi}{5}}\left(\begin{array}{ccc}
J & 2 & J \\
-J & 0 & J
\end{array}\right)\left\langle J\left\|\hat{T}^{(\mathrm{E} 2)}\right\| J\right\rangle,
$$

where use is made of the well known Wigner's 3-j symbol [55].

\section{A. Level-energy systematics}

Figure 3 displays the theoretical (a) and the experimental [56] (b) low-lying spectra as functions of the mass number. In the nuclei ${ }^{184-188} \mathrm{~Pb}$, the relative location of the $0_{2}^{+}$and $2_{1}^{+}$experimental levels is nicely reproduced. Our calculations reproduce the correct location in energy for these first excited $0^{+}$states, with the $0_{2}^{+}$level coming down as we approach the midshell nucleus ${ }^{186} \mathrm{~Pb}$ and becoming the lowest-energy one at ${ }^{186} \mathrm{~Pb}$ or ${ }^{188} \mathrm{~Pb}$. Both prolate and oblate minima become lowest in energy for these nuclei (see Fig. 1) and therefore the residual 


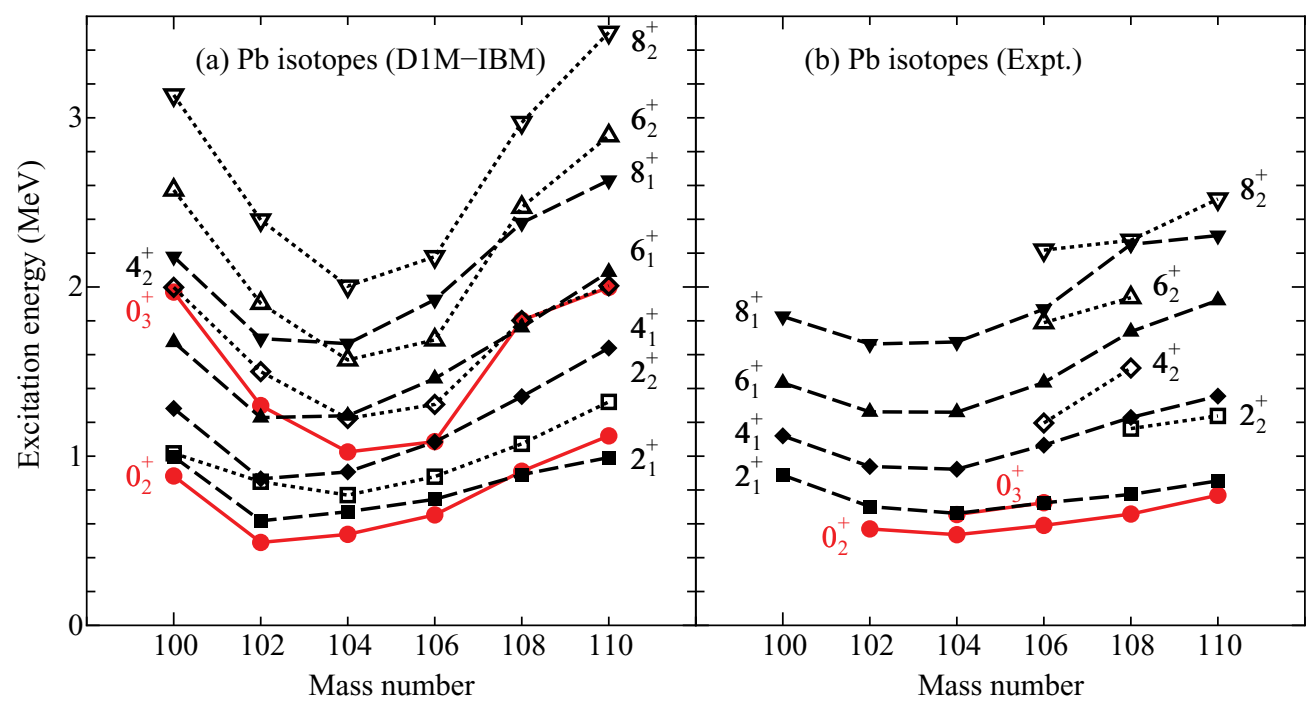

FIG. 3. (Color online) Level-energy systematics for ${ }^{182-192} \mathrm{~Pb}$ isotopes with mass number. Theoretical level energies resulting from the mapped IBM-2 Hamiltonian (a) are compared with the experimental (b) energies. The experimental data are taken from the ENSDF database [56]. To guide the eye, each point has been connected. Solid, dashed, and dotted lines stand for the lowest two excited $0^{+}$states, yrast states with $J \geqslant 2\left(2_{1}^{+}, 4_{1}^{+}, 6_{1}^{+}\right.$, and $\left.8_{1}^{+}\right)$and nonyrast states with $J \geqslant 2\left(2_{2}^{+}, 4_{2}^{+}, 6_{2}^{+}\right.$, and $\left.8_{2}^{+}\right)$, respectively. Note that the experimental $2_{1}^{+}$and $0_{3}^{+}$excitation energies for ${ }^{186} \mathrm{~Pb}\left({ }^{188} \mathrm{~Pb}\right)$ are $662(724)$ and $655(725) \mathrm{keV}$, respectively.

quadrupole-quadrupole correlation between neutron bosons and the intruder proton bosons becomes maximal, giving rise to these notably low-lying excited $0^{+}$states.

The comparison between our results and the few available data for the excitation energy of the $0_{3}^{+}$states reveals that our values overestimate the experimental ones. This could be due to the fact that in the considered isotopes the third lowest-energy minimum in the mean-field PESs appears higher than expected from the experimental point of view and also because of the level repulsion. Note that the parabolic behavior of the $0_{3}^{+}$levels with respect to midshell is in good agreement with the relative location of the three minima in the GognyPESs (see Fig. 1): the three minima are closest to each other around ${ }^{186} \mathrm{~Pb}$ while the second and third minima become less pronounced and only the spherical one remains as we approach the closed shells.

The present calculations also reproduce the parabolic tendency of states with angular momenta $J \geqslant 2$. The collectivity of the intruder configurations becomes stronger and, as a result, the intruder states with $J \geqslant 2$ become most compressed around the midshell. Nevertheless, the change in all the calculated energy levels, including the excited $0^{+}$ones, takes place faster as compared with the experimental trend. Let us also stress that, similar to the situation observed for the $0_{3}^{+}$ levels, the calculated nonyrast $2_{2}^{+}, 4_{2}^{+}, 6_{2}^{+}$, and $8_{2}^{+}$spectra are more stretched than the experimental ones.

\section{B. Structure of eigenfunctions}

To interpret the dominant component in the calculated excited states and the structure of the wave functions, we show in Table I the overlap probabilities of the basis states and the eigenfunctions corresponding to the three lowest-excited $0^{+}$ states for all the considered lead isotopes. In all the isotopes the $0_{1}^{+}$state corresponds to the spherical ground state with a nearly $100 \%$ dominance of the $0 \mathrm{p}-0 \mathrm{~h}$ configuration. For the nuclei ${ }^{182,184,186} \mathrm{~Pb}$, the first excited $0^{+}$state is comprised predominantly of the $4 \mathrm{p}-4 \mathrm{~h}$ configuration, which corresponds to the prolate minimum in Fig. 1. The extent of mixing between the $2 \mathrm{p}-2 \mathrm{~h}$ and the $4 \mathrm{p}-4 \mathrm{~h}$ configurations for the first excited $0^{+}$state becomes gradually stronger from ${ }^{182,184} \mathrm{~Pb}$ to ${ }^{186} \mathrm{~Pb}$, which correlates well with the finding in Fig. 1 that the oblate minimum becomes more significant from ${ }^{182,184} \mathrm{~Pb}$ to ${ }^{186} \mathrm{~Pb}$.

Experimentally both ${ }^{186,188} \mathrm{~Pb}$ are regarded as the most spectacular examples of shape coexistence in the $\mathrm{Pb}$ isotopic chain. In this case, one sees a stronger mixing between different configurations in the first and the second excited $0^{+}$states. The $0_{2}^{+}$state in ${ }^{186} \mathrm{~Pb}$ is more or less clearly of $4 \mathrm{p}-4 \mathrm{~h}$ character while the two intruder configurations are mixed for the $0_{2}^{+}$ state in ${ }^{188} \mathrm{~Pb}$. The earlier IBM-1 fitting calculation [17] suggested almost the same predominance of the $0_{2}^{+}$and the $\mathrm{O}_{3}^{+}$eigenfunctions while the three configurations appear to be more strongly mixed for ${ }^{186} \mathrm{~Pb}$. On the other hand, the

TABLE I. Fraction of each configuration in the lowest three $0^{+}$ states of the considered ${ }^{182-192} \mathrm{~Pb}$ isotopes (in \%).

\begin{tabular}{rcrrrrrc}
\hline \hline$J^{\pi}$ & Configurations & ${ }^{182} \mathrm{~Pb}$ & ${ }^{184} \mathrm{~Pb}$ & ${ }^{186} \mathrm{~Pb}$ & ${ }^{188} \mathrm{~Pb}$ & ${ }^{190} \mathrm{~Pb}$ & ${ }^{192} \mathrm{~Pb}$ \\
\hline \multirow{4}{*}{$0_{1}^{+}$} & 0p-0h & 100.0 & 99.8 & 99.7 & 98.6 & 99.7 & 99.6 \\
& 2p-2h & 0.0 & 0.2 & 0.2 & 1.3 & 0.3 & 0.4 \\
& 4p-4h & 0.0 & 0.0 & 0.1 & 0.1 & 0.0 & - \\
$0_{2}^{+}$ & 0p-0h & 0.0 & 0.1 & 0.1 & 1.4 & 0.5 & 0.5 \\
& 2p-2h & 9.0 & 14.6 & 24.3 & 65.5 & 92.8 & 99.5 \\
& 4p-4h & 91.0 & 85.3 & 75.6 & 33.1 & 6.7 & - \\
$0_{3}^{+}$ & 0p-0h & 34.1 & 0.9 & 0.5 & 0.7 & 98.7 & 93.2 \\
& 2p-2h & 41.2 & 67.9 & 67.5 & 36.1 & 1.2 & 6.8 \\
& $4 \mathrm{p}-4 \mathrm{~h}$ & 24.7 & 31.2 & 32.0 & 63.2 & 0.1 & - \\
\hline \hline
\end{tabular}




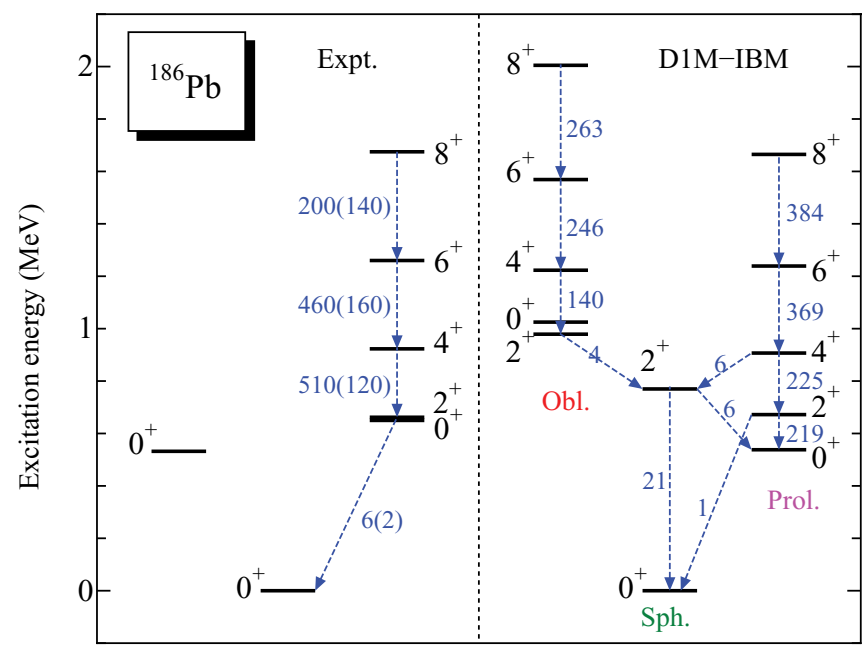

FIG. 4. (Color online) Experimental and calculated energy spectra and $B(\mathrm{E} 2)$ transition rates [in Weisskopf units (W.u.)] for the ${ }^{186} \mathrm{~Pb}$ nucleus. Experimental energies and $B(\mathrm{E} 2)$ values are taken from [56,57]. In the plot the experimental $0_{3}^{+}$and $2_{1}^{+}$look nearly degenerated, but their excitation energies are 650 and $662 \mathrm{keV}$, respectively. The experimental $B(\mathrm{E} 2)$ of $510(120)$ and 6 (2) (in W.u.) correspond to the $4_{1}^{+} \rightarrow 2_{1}^{+}$and $2_{1}^{+} \rightarrow 0_{1}^{+}$transitions, respectively.

present results for ${ }^{186} \mathrm{~Pb}$ seem to be consistent with the ones obtained within the symmetry projected GCM approximation based on both the Skyrme-SLy6 [32] and Gogny-D1S [35] EDFs. In such studies [32,35], the collective wave function for the $0_{2}^{+}\left(0_{3}^{+}\right)$excited state is peaked on the prolate (oblate) side. We also find that our results for the nucleus ${ }^{188} \mathrm{~Pb}$ in Table I are qualitatively consistent with the ones of previous symmetry projected GCM studies [33,35] where collective wave functions strongly peaked at the oblate and prolate sides have also been predicted. For the nuclei ${ }^{190,192} \mathrm{~Pb}$, there is almost no mixing between the different configurations for the three $0^{+}$states. In fact, the fraction of the $4 \mathrm{p}-4 \mathrm{~h}$ configuration is too small for them.

\section{Level scheme: ${ }^{186,188} \mathrm{~Pb}$ nuclei}

In this section, we discuss in more detail the results obtained for the isotopes ${ }^{186} \mathrm{~Pb}$ and ${ }^{188} \mathrm{~Pb}$, which are the most distinct cases of shape coexistence in the considered chain. We compare in Figs. 4 and 5 our theoretical and the experimental energy levels and transition rates for these nuclei. The assignment of the calculated excited state to each band is done according to the predominance of a given configuration in the corresponding eigenstate and the E2 transition strength that exhibits a clear collectivity.

For ${ }^{186} \mathrm{~Pb}$, in Fig. 4, the calculated first excited $0_{2}^{+}$state, predicted to be predominantly prolate, is quite close to the experimental value. From the experimental point of view, such a state has been identified [2] as the oblate bandhead. On the other hand, our result in Fig. 4 is consistent with earlier predictions for the same nucleus within the symmetry projected GCM approximation based on the functionals Skyrme-SLy6 [32] and Gogny-D1S [35]. Actually, as seen from Table I, the 4p-4h

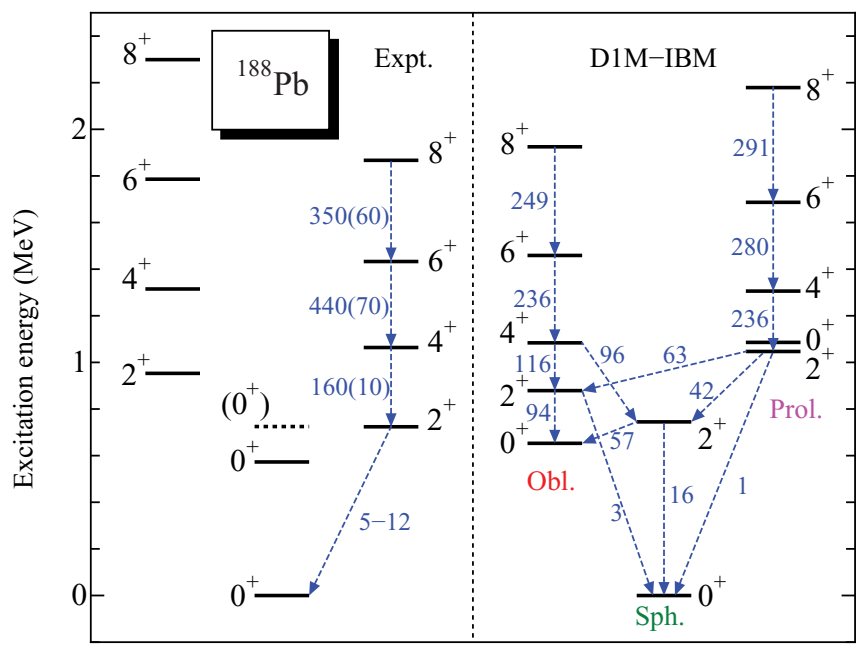

FIG. 5. (Color online) Same as Fig. 4, but for the ${ }^{188} \mathrm{~Pb}$ nucleus. Note that the theoretical $0_{3}^{+}$and $2_{3}^{+}$excitation energies are 1.086 and $1.047 \mathrm{MeV}$, respectively. The following theoretical $B(\mathrm{E} 2)$ in the right panel are listed here to help identify the corresponding transition in the plot: $B\left(\mathrm{E} 2 ; 4_{2}^{+} \rightarrow 2_{3}^{+}\right)=236, B\left(\mathrm{E} 2 ; 2_{3}^{+} \rightarrow 2_{2}^{+}\right)=63, B\left(\mathrm{E} 2 ; 2_{3}^{+} \rightarrow\right.$ $\left.2_{1}^{+}\right)=42$, and $B\left(\mathrm{E} 2 ; 2_{3}^{+} \rightarrow 0_{1}^{+}\right)=1$ (in W.u.).

(prolate in the present IBM framework) component dominates $75.6 \%$ of the $0_{2}^{+}$state. A strong collective energy pattern is also predicted for this prolate band, with the ratio $\Delta E_{4_{1}^{+}} / \Delta E_{2_{1}^{+}}=$ 2.75 . The $B(\mathrm{E} 2)$ transitions among the members of this prolate band exhibit a collective behavior while the $2^{+} \rightarrow 0^{+}$E2 transition is very weak in the spherical band. Concerning the oblate band, the theoretical $0_{3}^{+}$excitation energy overestimates the experimental one. Note that, experimentally, this $0_{3}^{+}$state is recognized as the prolate bandhead [2]. The experimental $2_{1}^{+}$ and $0_{3}^{+}$levels look nearly degenerated, and so does the present calculation except that the $2_{3}^{+}$level lies slightly below the $0_{3}^{+}$ level since the mixing between the two intruder configurations may be too strong.

One notices from Fig. 5 that our model provides a similar level of quality in the description of the isotope ${ }^{188} \mathrm{~Pb}$. Although the calculated excitation energy for the $0_{3}^{+}$state is a bit high, the calculated $\mathrm{O}_{2}^{+}$state lies close to the experimental one. The present study also suggests that the $0_{2}^{+}$and the $0_{3}^{+}$levels correspond to oblate and prolate configurations, respectively, which is consistent with symmetry projected GCM calculations based on the Gogny-D1S EDF [35]. Nevertheless, the first and the second excited $0^{+}$states are experimentally [56] interpreted as the prolate and the oblate bandheads, respectively. Moreover, the present study suggests a pronounced collective pattern for both the prolate $(4 p-4 h)$ and the oblate $(2 p-2 h)$ bands, and supports the experimental evidence for the strong E2 transition pattern in the band comprised of $2_{1}^{+}, 4_{1}^{+}, 6_{1}^{+}$, and $8_{1}^{+}$states. In our calculations, the two intruder $0^{+}$levels are rather close in energy, compared to the case of ${ }^{186} \mathrm{~Pb}$. In fact, among all the considered nuclei, the prolate-oblate energy difference obtained from the Gogny-D1M PESs in Fig. 1 reaches its lowest value for ${ }^{188} \mathrm{~Pb}$. Due to the level repulsion, however, the excitation energy of the $0_{3}^{+}$state is larger than the energy difference between the spherical and prolate minima of the corresponding HFB PES in Fig. 1. 


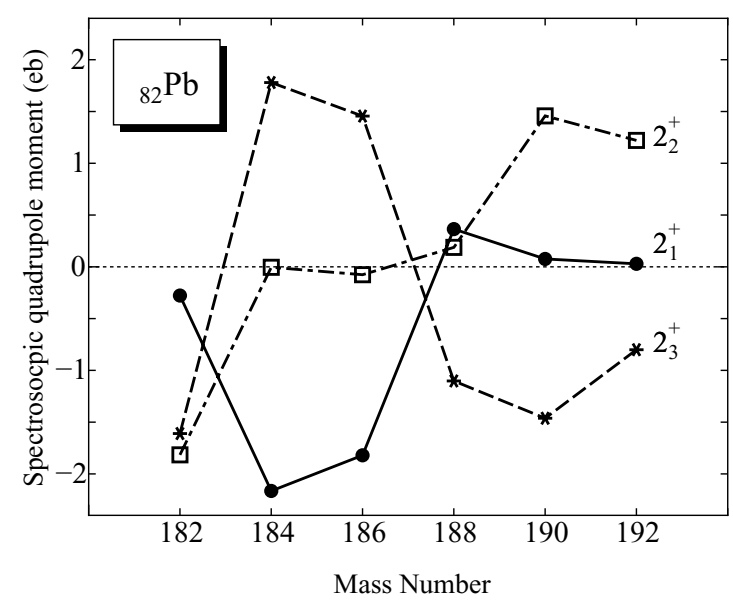

FIG. 6. Calculated spectroscopic quadrupole moments $Q^{(s)}$ for the lowest three excited $2^{+}$states of the considered $\mathrm{Pb}$ nuclei as functions of mass number. Solid, dashed, and dot-dashed lines connect the calculated $Q^{(s)}$ values for $2_{1}^{+}, 2_{2}^{+}$, and $2_{3}^{+}$states, respectively.

\section{Spectroscopic quadrupole moment}

The spectroscopic quadrupole moment $Q^{(s)}$, computed according to Eq. (11), is shown in Fig. 6 as a function of the mass number for the three lowest $2^{+}$excited states of the considered lead isotopes. In the case of ${ }^{182} \mathrm{~Pb}, Q^{(s)}\left(2_{1}^{+}\right) \approx 0 e \mathrm{~b}$ and $Q^{(s)}\left(2_{2}^{+}\right) \approx Q^{(s)}\left(2_{3}^{+}\right) \approx-2 e \mathrm{~b}$, reflecting the spherical and prolate character of the $2_{1}^{+}$and $2_{2}^{+}, 2_{3}^{+}$states, respectively. The microscopic and the mapped PESs for this nucleus (see Fig. 1) suggest a global spherical minimum and a welldeveloped prolate deformation. The two nonyrast $2^{+}$states should originate from such a pronounced prolate minimum. For ${ }^{184,186} \mathrm{~Pb}$, both the HFB and the mapped PESs in Fig. 1 indicate the development of triple coexistence. The trend of the considered quadrupole moment changes accordingly. The $Q^{(s)}\left(2_{1}^{+}\right)\left(Q^{(s)}\left(2_{3}^{+}\right)\right)$value is nearly $-2(+1.5) e \mathrm{~b}$, suggesting that this state is prolate (oblate). From Fig. 4, one realizes that the prolate band consisting of the $0_{2}^{+}, 2_{1}^{+}, 4_{1}^{+}, 6_{1}^{+}$, and $8_{1}^{+}$states comes down in energy. On the other hand, our calculations suggest that the third band in ${ }^{186} \mathrm{~Pb}$, comprised of the $2_{3}^{+}$, $0_{3}^{+}, 4_{2}^{+}, 6_{2}^{+}$, and $8_{2}^{+}$states, originates from the $2 \mathrm{p}-2 \mathrm{~h}$ oblate configuration. Note that the quadrupole moment for the $2_{3}^{+}$ state is positive. The same arguments apply to the nucleus ${ }^{184} \mathrm{~Pb}$.

A change in the spectroscopic quadrupole moments from ${ }^{186} \mathrm{~Pb}$ to ${ }^{188} \mathrm{~Pb}$ is also apparent from Fig. 6. In ${ }^{188} \mathrm{~Pb}, Q^{(s)}\left(2_{2}^{+}\right)=$ $0.19 e \mathrm{~b}$ while $Q^{(s)}\left(2_{1}^{+}\right)=0.36 e \mathrm{~b}$. On the other hand, the $Q^{(s)}\left(2_{3}^{+}\right)$value becomes negative $(=-1.10 e \mathrm{~b})$. As can be observed from the level scheme displayed in Fig. 5, the $2_{1}^{+}$state consists exclusively of the regular (spherical) configuration. The band consisting of the $0_{2}^{+}, 2_{2}^{+}, 4_{1}^{+}, 6_{1}^{+}$, and $8_{1}^{+}$states emerges with predominant $2 \mathrm{p}-2 \mathrm{~h}$ oblate character while the one composed of the $0_{3}^{+}, 2_{3}^{+}, 4_{2}^{+}, 6_{2}^{+}$, and $8_{2}^{+}$states emerges with $4 \mathrm{p}-4 \mathrm{~h}$ prolate character. It should be noted, however, that the spectroscopic quadrupole moment $Q^{(s)}\left(2_{2}^{+}\right)$for the $2_{2}^{+}$state, assigned to the oblate band due to its stronger E2 transition to the $0_{2}^{+}$state, is quite close to the $Q^{(s)}\left(2_{1}^{+}\right)$ value. This is mainly due to the fact that the mixing between the different configurations is too strong for these two $2^{+}$ states: for the $2_{1}^{+}\left(2_{2}^{+}\right)$state, $58(41), 32(38)$, and $10(21) \%$ of its eigenfunction is composed of spherical $0 \mathrm{p}-0 \mathrm{~h}$, oblate $2 \mathrm{p}-2 \mathrm{~h}$, and prolate $4 \mathrm{p}-4 \mathrm{~h}$ configurations, respectively. The value $Q^{(s)}\left(2_{3}^{+}\right)=-1.10$ e b reflects a more clear prolate character, as the three configurations are less strongly mixed in this $2_{3}^{+}$state: 2,38 , and $60 \%$ of the eigenfunction is composed of $0 \mathrm{p}-0 \mathrm{~h}, 2 \mathrm{p}-2 \mathrm{~h}$, and $4 \mathrm{p}-4 \mathrm{~h}$ configurations, respectively.

For both ${ }^{190,192} \mathrm{~Pb}$, we obtain that $Q^{(s)}\left(2_{1}^{+}\right)$is close to zero so that the $2_{1}^{+}$state is supposed to be of spherical character. Our result seems to support the fact that the $2_{2}^{+}$state is composed predominantly of the $2 \mathrm{p}-2 \mathrm{~h}$ oblate configuration. This result agrees well with the corresponding PESs, shown in Fig. 1, for which the oblate minimum lies much lower, compared to ${ }^{182-188} \mathrm{~Pb}$, than the prolate one. Note also that $Q^{(s)}\left(2_{3}^{+}\right)<0$ for ${ }^{190,192} \mathrm{~Pb}$, implying that the $2_{3}^{+}$state is prolate.

\section{SUMMARY}

To summarize, the emergence and evolution of the shape coexistence in the neutron-deficient lead isotopes have been investigated within the configuration mixing IBM model with parameters extracted solely from a mapping of the mean-field PESs obtained with the Gogny-D1M EDF. The diagonalization of the IBM Hamiltonian provides energy levels as well as transition rates between the excited states. It is important to emphasize that, although the IBM configuration mixing model contains many parameters, they can be determined unambiguously by relating the IBM PES for each configuration to the corresponding mean-field deformation minimum in the microscopic PES. No additional adjustment to experimental data is required. A potential difficulty and uncertainty of the fully consistent mapping concerning the offset energy $\Delta$ has been addressed and possible remedies for it have been discussed.

The considered lead nuclei present the most spectacular example of the coexistence of spherical, oblate, and prolate equilibrium shapes. The relative locations of the three associated $0^{+}$states were reproduced. In one of the most stringent tests, the ${ }^{186} \mathrm{~Pb}$ nucleus, the present calculation suggested that the $0_{2}^{+}$and the $0_{3}^{+}$states are predominantly of prolate $(4 p-4 h)$ and oblate $(2 p-2 h)$ nature, respectively. For the ${ }^{188} \mathrm{~Pb}$ nucleus, another typical example with more available experimental data to compare with, the present work predicts the oblate bandhead as the first excited $0^{+}$ state and the prolate band as the second excited $0^{+}$state. The calculated E2 transition pattern, albeit the quantitative deviation of the interband transitions from the experimental data, provides indications of strong collectivity for the relevant prolate and oblate shapes. The experimental level-energy systematics is well reproduced by our calculations. The study of the prolate-oblate dynamics has been complemented by looking at the spectroscopic quadrupole moment. Its value for different configurations and nuclei is consistent with the implications of other quantities and the suggestions of the mean-field microscopic calculations.

Using the proposed methodology, many new research directions concerning complex shape dynamics are opened up. A 
possible application would be to analyze neighboring isotopic chains, mercury, polonium, and platinum isotopes. In particular, the study in the platinum isotopes will help to disentangle if the single configuration is the appropriate picture to describe those isotopes (see, e.g., $[19,43]$ and references therein). Other mass regions, including neutron-deficient krypton, selenium, and germanium isotopes and neutron-rich krypton, strontium, and zirconium isotopes, which are also known as regions of shape coexistence [4], would be a potential target.

The predictive power endowed to the model by the microscopic input makes possible the application of the present methodology to the study of exotic nuclei like the ones that will be experimentally accessible in the near future.

\section{ACKNOWLEDGMENTS}

The authors would like to thank J. Jolie and R. V. Jolos for valuable discussions and T. Otsuka for his continuous interest in this work. K.N. acknowledges the support of the Japan Society for the Promotion of Science Postdoctoral Fellowships for Research Abroad. L.M.R. acknowledges the support of Ministerio de Economía y Competitividad through Grants No. FPA2009-08958 and No. FIS2009-07277, as well as the Consolider-Ingenio 2010 program CPAN CSD2007-00042 and MULTIDARK CSD2009-00064. N.S. acknowledges the support of Strategic Programs for Innovative Research field 5, Ministry of Education, Culture, Sports, Science, and Technology, Japan.
[1] J. Wood, K. Heyde, W. Nazarewicz, M. Huyse, and P. van Duppen, Phys. Rep. 215, 101 (1992).

[2] A. N. Andreyev et al., Nature (London) 405, 430 (2000).

[3] R. Julin, K. Helariutta, and M. Muikku, J. Phys. G 27, R109 (2001).

[4] K. Heyde and J. L. Wood, Rev. Mod. Phys. 83, 1467 (2011).

[5] P. Van Duppen, E. Coenen, K. Deneffe, M. Huyse, K. Heyde, and P. Van Isacker, Phys. Rev. Lett. 52, 1974 (1984).

[6] K. Heyde, P. V. Isacker, R. Casten, and J. Wood, Phys. Lett. B 155, 303 (1985).

[7] K. Heyde, J. Jolie, J. Moreau, J. Ryckebusch, M. Waroquier, P. V. Duppen, M. Huyse, and J. Wood, Nucl. Phys. A 466, 189 (1987).

[8] P. Federman and S. Pittel, Phys. Lett. B 69, 385 (1977).

[9] K. Heyde, C. De Coster, J. Jolie, and J. L. Wood, Phys. Rev. C 46, 541 (1992).

[10] F. Iachello and A. Arima, The Interacting Boson Model (Cambridge University Press, Cambridge, 1987).

[11] A. Arima, T. Otsuka, F. Iachello, and I. Talmi, Phys. Lett. B 66, 205 (1977)

[12] T. Otsuka, A. Arima, F. Iachello, and I. Talmi, Phys. Lett. B 76, 139 (1978).

[13] T. Otsuka, A. Arima, and F. Iachello, Nucl. Phys. A 309, 1 (1978).

[14] P. D. Duval and B. R. Barrett, Phys. Lett. B 100, 223 (1981).

[15] P. D. Duval and B. R. Barrett, Nucl. Phys. A 376, 213 (1982).

[16] A. F. Barfield, B. R. Barrett, K. A. Sage, and P. D. Duval, Z. Phys. A 311, 205 (1983).

[17] R. Fossion, K. Heyde, G. Thiamova, and P. Van Isacker, Phys. Rev. C 67, 024306 (2003).

[18] V. Hellemans, S. De Baerdemacker, and K. Heyde, Phys. Rev. C 77, 064324 (2008).

[19] J. E. García-Ramos, V. Hellemans, and K. Heyde, Phys. Rev. C 84, 014331 (2011).

[20] C. D. Coster, K. Heyde, B. Decroix, P. V. Isacker, J. Jolie, H. Lehmann, and J. Wood, Nucl. Phys. A 600, 251 (1996).

[21] H. Lehmann, J. Jolie, C. D. Coster, B. Decroix, K. Heyde, and J. Wood, Nucl. Phys. A 621, 767 (1997).

[22] A. Frank, P. Van Isacker, and C. E. Vargas, Phys. Rev. C 69, 034323 (2004)

[23] A. Frank, P. Van Isacker, and F. Iachello, Phys. Rev. C 73, 061302 (2006).

[24] I. O. Morales, A. Frank, C. E. Vargas, and P. Van Isacker, Phys. Rev. C 78, 024303 (2008).
[25] M. Bender, P.-H. Heenen, and P.-G. Reinhard, Rev. Mod. Phys. 75, 121 (2003).

[26] J. Decharge, M. Girod, and D. Gogny, Phys. Lett. B 55, 361 (1975).

[27] D. Vretenar, A. Afanasjev, G. Lalazissis, and P. Ring, Phys. Rep. 409, 101 (2005).

[28] T. Nikšić, D. Vretenar, and P. Ring, Prog. Part. Nucl. Phys. 66, 519 (2011).

[29] P.-G. Reinhard, D. J. Dean, W. Nazarewicz, J. Dobaczewski, J. A. Maruhn, and M. R. Strayer, Phys. Rev. C 60, 014316 (1999).

[30] T. Nikšić, D. Vretenar, P. Ring, and G. A. Lalazissis, Phys. Rev. C 65, 054320 (2002).

[31] W. Nazarewicz, Phys. Lett. B 305, 195 (1993).

[32] T. Duguet, M. Bender, P. Bonche, and P.-H. Heenen, Phys. Lett. B 559, 201 (2003).

[33] M. Bender, P. Bonche, T. Duguet, and P.-H. Heenen, Phys. Rev. C 69, 064303 (2004).

[34] R. Chasman, J. Egido, and L. Robledo, Phys. Lett. B 513, 325 (2001).

[35] R. R. Rodríguez-Guzmán, J. L. Egido, and L. M. Robledo, Phys. Rev. C 69, 054319 (2004).

[36] J. L. Egido, L. M. Robledo, and R. R. Rodríguez-Guzmán, Phys. Rev. Lett. 93, 082502 (2004).

[37] J.-P. Delaroche et al. Phys. Rev. C 81, 014303 (2010).

[38] Z. P. Li, T. Nikšić, D. Vretenar, and J. Meng, Phys. Rev. C 81, 034316 (2010).

[39] J. P. Delaroche et al., Phys. Rev. C 50, 2332 (1994).

[40] K. Nomura, N. Shimizu, and T. Otsuka, Phys. Rev. Lett. 101, 142501 (2008).

[41] K. Nomura, N. Shimizu, and T. Otsuka, Phys. Rev. C 81, 044307 (2010).

[42] K. Nomura, T. Otsuka, N. Shimizu, and L. Guo, Phys. Rev. C 83, 041302 (2011).

[43] K. Nomura, T. Otsuka, R. Rodríguez-Guzmán, L. M. Robledo, and P. Sarriguren, Phys. Rev. C 83, 014309 (2011).

[44] K. Nomura, N. Shimizu, D. Vretenar, T. Nikšić, and T. Otsuka, Phys. Rev. Lett. 108, 132501 (2012).

[45] S. Goriely, S. Hilaire, M. Girod, and S. Péru, Phys. Rev. Lett. 102, 242501 (2009).

[46] R. Rodríguez-Guzmán, P. Sarriguren, L. Robledo, and S. PerezMartin, Phys. Lett. B 691, 202 (2010).

[47] R. Rodriguez-Guzman, P. Sarriguren, and L. M. Robledo, Phys. Rev. C 82, 044318 (2010). 
[48] R. Rodríguez-Guzmán, P. Sarriguren, and L. M. Robledo, Phys. Rev. C 82, 061302 (2010).

[49] J. Berger, M. Girod, and D. Gogny, Nucl. Phys. A 428, 23 (1984).

[50] A. Bohr and B. M. Mottelsson, Nuclear Structure (Benjamin, New York, 1975), Vol. 2.

[51] R. Rodríguez-Guzmán, P. Sarriguren, L. M. Robledo, and J. E. García-Ramos, Phys. Rev. C 81, 024310 (2010).

[52] J. N. Ginocchio and M. W. Kirson, Nucl. Phys. A 350, 31 (1980).
[53] T. Mizusaki and T. Otsuka, Prog. Theor. Phys. Suppl. 125, 97 (1996).

[54] D. D. Warner and R. F. Casten, Phys. Rev. C 28, 1798 (1983).

[55] D. Varshalovich, A. Moskalev, and V. Khersonski, Quantum Theory of Angular Momentum: Irreducible Tensors, Spherical Harmonics, Vector Coupling Coefficients, 3nj Symbols (World Scientific, Singapore, 1988).

[56] Brookhaven National Nuclear Data Center, http://www.nndc. bnl.gov (ENSDF).

[57] T. Grahn et al., Phys. Rev. Lett. 97, 062501 (2006). 